\title{
DDSS: Dynamic Decision Support System for Elderly
}

\author{
Asad Masood Khattak ${ }^{1}$, Zeeshan Pervez ${ }^{1}$, Manhyung Han ${ }^{1}$, Chris Nugent ${ }^{2}$, Sungyoung Lee ${ }^{1}$ \\ ${ }^{1}$ Department of Computer Engineering, Kyung Hee University, Korea, \\ \{asad.masood,zeeshan,smiley,sylee\}@oslab.ac.kr \\ ${ }^{2}$ School of Computing and Mathematics, University of Ulster, UK, \\ cd.nugent@ulster.ac.uk
}

\begin{abstract}
To provide robust healthcare services and personalized recommendations details relating to a patient's daily life activities, profile information, and personal experience is of vital importance. This paper focuses on improvement in general health status of elderly patients through the use of an innovative service which align dietary intake with activity information. Personalized healthcare services based on the patient's activities of daily living and their shared experience, are provided as outputs. A knowledge driven approach has been used where all the daily life activities, social interactions, and profile information are modeled in an ontology. The semantic context is exploited that enables fine-grained situation analysis for recommendation of personalized services and decision support. Preliminary experimental results for the dynamic nature of the systems and its corresponding personalized recommendations have been found to be encouraging.
\end{abstract}

\section{Introduction}

Effects of ageing on the population are causing huge social and economic challenges. This is currently affecting all countries on a global scale and is resulting in producing a wide range of changes in health and social care policies. As recognized by the European Commission more than $20 \%$ of Europeans will be 65 or over by 2025 . A financially viable and sustainable supporting mechanism for the issues associated with ageing is therefore required.

According to World Health Organization (WHO), one of the groups that can benefit the most from regularly performed exercise is the elderly [15]. The benefits of exercise have been associated with the prevention and treatment of many chronic and age related conditions for example hypertension, heart disease and even cognitive decline [4]. In addition to exercise, the effects of maintaining a healthy diet have also been recognized $[4,12]$. Nevertheless, there is a general lack of nutritional knowledge within the population. The use of pervasive technologies could provide the means of encouraging and promoting healthier food preparation [12]. A further component in the wellness paradigm in addition to exercise and nutrition is that of social integration and interaction. According to the WHO's Active Ageing program, active ageing highlights the importance of social interaction throughout the life course [14].

In today's society, people are interested in their improved healthcare. Hence, a challenge exists to both provide and maintain the quality and availability of healthcare services with a minimum cost [9]. Various wireless technologies have been used for improved healthcare services. CodeBlue [6] supports physicians and nurses to monitor patients. Reminder based systems which have primarily focused on plan-based approaches to decide how and when to prompt subjects effectively [11] in addition to offering location-based reminders [13]. Nevertheless, the context for the delivery of reminders involves more information than simply location or time. HYCARE [3] system takes context in consideration and develops a schedule for reminder based services with a goal of avoiding possible conflicts. In [16], an ontology is used to incorporate context related information for intelligent processing; however, the system developed was more of a homecare system rather than a healthcare system. Work presented in [1] details an ontology based reminder system; incorporates rules for manipulating the recognized activities of the elderly. In [10], realtime activities are recognized using diverse sensors are used for situation analysis. Research in [5] focused on the social interaction of patients. Based on the experiences shared, it generates intelligent service recommendations and eliminates their isolation. 


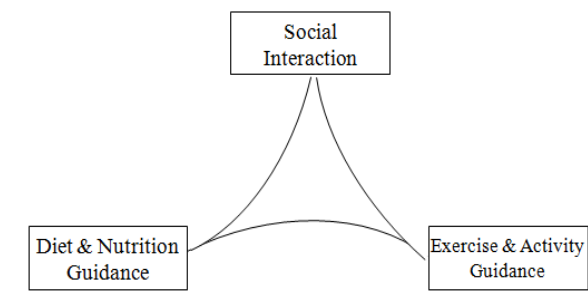

Figure 1, Shows the main SWELL component-wise interaction model.

Existing systems aiming to offer a form of support are mostly based on one input modality and in some cases use imperfect contextual information [7]. These solutions subsequently do not consider the integration of activity and social interaction for the purposes of service recommendation. Existing solutions may be considered as static and are not adaptive with the environment and dynamic nature of the patient's behavior and do not consider domain knowledge. To facilitate achieving the objective of dynamic decision support and provision of services to make suggestions for dietary intake based on a patient's activities and social interaction, the concept of Social Wellness for the Elderly (SWELL) is introduced in this research. It offers improvement in the general health status of the elderly population through the use of an innovative service to align dietary intake with activity related information which is promoted through a social network facilitated by contemporary Web 2.0 technologies (refer to Figure 1).

The Dynamic Decision Support System (DDSS) of the SWELL is the main focus of this research. DDSS is deployed on a Cloud Server and actively listens to its outer world agent (Smart Phone) for patient activities and instances of social interaction. Moreover, the DDSS uses this information for appropriate recommendations for physical activities and changes in dietary and nutritional intakes. Any change in the user's behavior is detected using activity recognition processes. Changes in the user's social interaction are reported automatically and along with behavioral changes are used as the basis for recommendations which are based on the updated user preferences. This concept supports the migration of patient information to/from third party systems and the proposed DDSS Knowledge Repository (KR). The KR is based on an ontology where patient information is formally represented and updated dynamically. The DDSS has been tested for its dynamic KR and service provisioning. The preliminary experimental results for recommendation and dynamic change management have been very encouraging.

This remainder of this paper is presented as follows: Section 2 briefly describes the SWELL system. Section 3 presents the proposed DDSS in detail. Section 4 provides a discussion on the proposed scheme's experimental results. Finally we conclude our Discussion in Section 5.

\section{Social Wellness for EIderly (SWELL)}

This section briefly describes the component-wise architecture and workings of SWELL. SWELL is essentially composed of three main components (refer to Figure 2), namely a Pervasive Agent (PA), Secure Cloud Storage (SCS), and Dynamic Decision Support System (DDSS). The DDSS component is the focus of this research paper, hence this section will briefly introduce the concepts related to the PA and SCS.

The PA (Smart Phone) used by the patients provides the facility of recognizing the user activities through the use of embedded sensors and wirelessly connected medical devices. Nine different sensors (GPS, Accelerometer, Camera, Proximity, Light, Time, Gyroscope, Bluetooth, and MIC) of the PA are used. Patient activities and dietary intakes are also captured using the PA. Levels of social interaction using SMS or community chat on the PA along with ECG, Heart Rate, Blood Pressure and Glucose Levels collected by

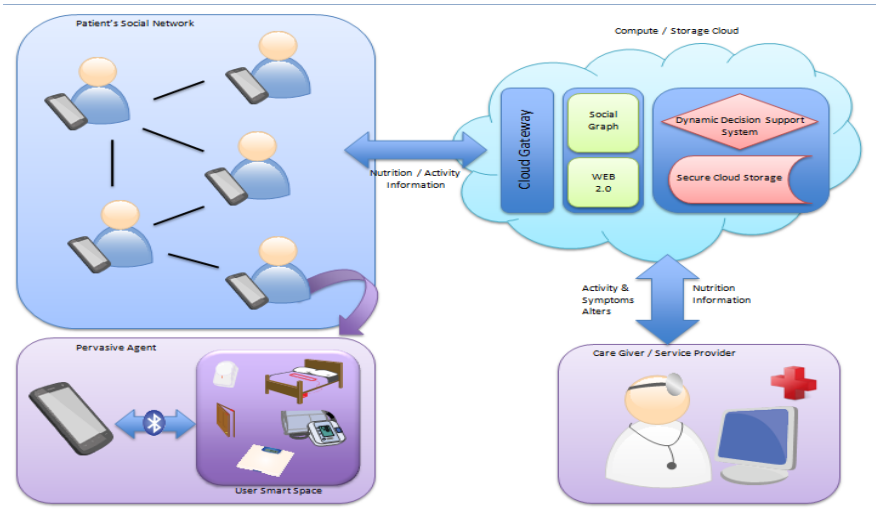

Figure 2, The overall architecture of SWELL. 
the PA whilst communicating with healthcare devices are all forwarded to the DDSS which is hosted on a Cloud server. Following manipulation, it generates personalized recommendations for nutritional and dietary intakes, physical activities and futuristic social interactions and provides these to the patients via PA.

SWELL engages all stakeholders involved in dietary, activity and social interaction related activities and promotes the concept of social sharing. Patients have the ability to share their activity and health information with other stakeholders (i.e., patients, relatives, friends, and medical doctors) in their social graph. The participants themselves set the privacy features provided in SWELL hence providing them with full empowerment over their data. The SCS achieves data privacy while facilitating seamless data sharing amongst all of the involved users. It works as a secure bridge between the data on the cloud and the user. Its main responsibility is to insure that the access control policies are never compromised and legitimate users may always have access to the outsourced data. To achieve this, digital certificates of legitimate users are maintained along with identity assertions used to access the data while preserving the privacy.

\section{Dynamic Decision Support System}

Activities of daily living and social information are used for generating intelligent personalized nutritional and dietary recommendations and social connections using the DDSS (refer to Figure 3). The DDSS communicates with the PA to receive its input and also produces the results back to the PA. It is composed of four main components as discussed below.

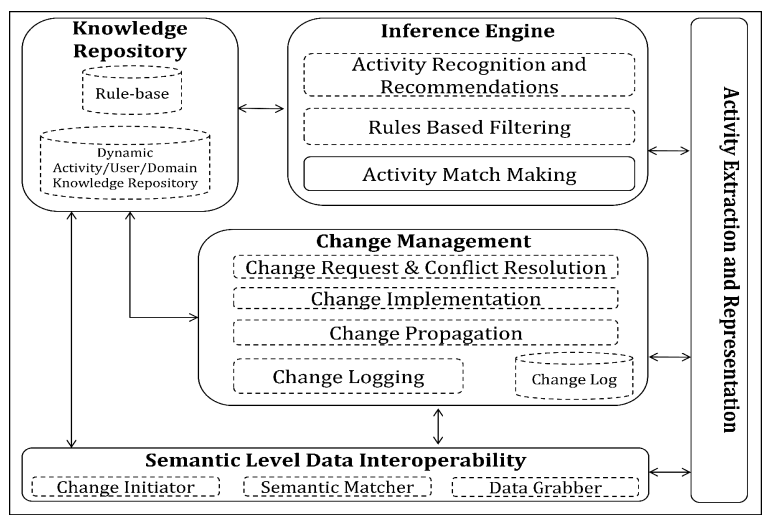

Figure 3, Architecture of the Dynamic Decision Support System (DDSS).

\subsection{Knowledge Repository}

The KR serves as backbone for the DDSS. It contains the evolving activity information, social interaction, patient profile information, and expert knowledge on the nutritional and dietary intakes. The information in the KR is modeled using an ontology. The knowledge in the Dynamic Activity Repository (DAR) is semantically linked (refer to Figure 4) for subsequent processing using the Inference Engine (IE). Following axiom is the representation of the proposed abstract model for representing knowledge in DAR.

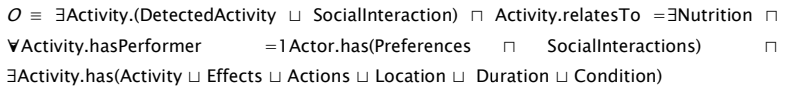

Once inference is completed on the information available from the DAR and PA, the inferred knowledge is filtered using the expert's (nutritionist) knowledge incorporated in rules (stored in Rule-base). This generates personalized recommendations for a patient's daily life dietary intakes. Both the DAR and Rule-base are dynamic in nature and evolve as the system learns more about the patients or environment or with the evolving domain knowledge.

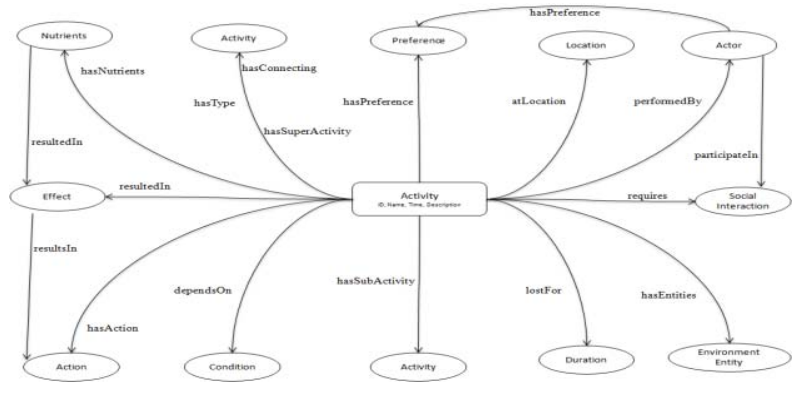

Figure 4, Activity, actor, and effects relationship modeling in the ontology for representation of information in the Knowledge Repository based on the concept presented in [1].

\subsection{Activity Extraction and Representation}

This module is the gateway of the DDSS to the PA. It accepts all the communication from the PA and then models it using the KR structure. Any communication with this module is termed as activity, including instances of social interaction. Social interaction is text mined and the subsequent activity information is extracted from it. All activities are stored in the KR. Any new migrating patient information from/to another system also passes through this module. The extracted activity is represented (refer to Figure 5) in the proposed ontological structure. Migrating activity/patient information is passed to the Semantic Level Data Interoperability (SLDI); change in existing knowledge is passed to the Change Management (CM) component and any newly reported activity is passed to the IE for the generation of recommendations. 


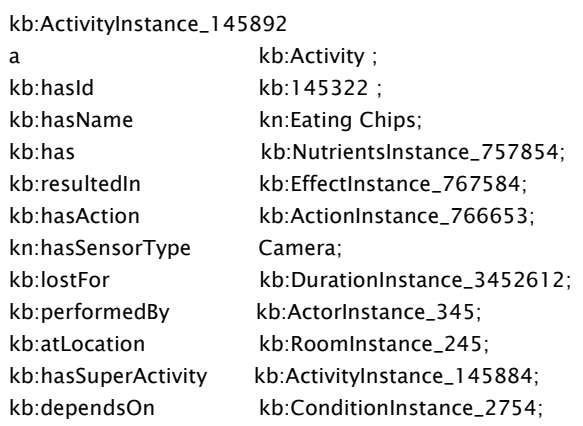

Figure 5, Ontological representation of activity in N3 notation.

\subsection{Inference Engine}

The IE is the brain of the DDSS. It is dedicated to performing the reasoning tasks and generating recommendations. The IE involves KR for situation analysis. Any newly detected activity is matched with knowledge from the DAR. The Rule-base is then used to filter the reasoned knowledge and to make appropriate recommendations for social interactions, nutrition and dietary intakes, and forms of physical activities. A two phase algorithm (refer to Algorithm 1) has been devised for the inference that uses Pellet as a reasoning engine to work on ontological data.

Algorithm RecommendationService(Process Patient's Activities):

This algorithm predefines matching threshold of activity to $\psi=0.70$. Input: Dynamic Activity ontology $O$ contains domain knowledge and patient's profile information. Instance of newly detected activity

$A, A \in O$. Decision rules $R$, used for decision making.

Output: Nutrition intake, social interaction and Activity recommendations.

1. $/ *$ Receive detected activities from Personal Agent */

2. $\operatorname{get} \operatorname{Activity}(A)$

3. $/ *$ Parse ontology for the knowledge, calculate semantic affinity $* /$

4. $O \leftarrow \operatorname{parse}(O)$

5. $O_{\text {sub }} \leftarrow \operatorname{semantic}$ Affinity $(A, O, \psi)$

6. $/ *$ Read rules from the Rule Base maintain in an array $\mathrm{R} * /$

7. $R[] \leftarrow$ readRules(Rulesbase)

8. /* Start recommendation process using forward chaining */

9. foreach $R_{\Delta} \in R[]$ do

10. $/ *$ Process for recommendation generation */

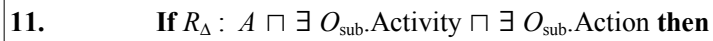

12. $/ *$ Store the recommendations in an array */

13. $\operatorname{RecomArr}[] \leftarrow\left\{\mathrm{x} \mid<R_{\Delta}, \mathrm{x}>\right.$ Action $\}$

14. else

15. $/ *$ Process for higher level activity */

16. HA[] $\leftarrow O \sqcap\{\mathrm{x} \mid<A, \mathrm{x}>$ hasAssociation $\}$

$17 . \quad$ EndIf

18. EndLoop

19. $/ *$ Execute valued recommendations and store higher activities*/

20. execute $(\operatorname{RecomArr}[\psi])$

21. $O \leftarrow O+H A[\psi]$

22. End

Algorithm-1, For activity manipulation and recommendations

\subsection{Semantic Level Data Interoperability}

The SLDI component calculates the semantic affinity for the incoming migrating information against the existing information in the DAR. Based on similarity threshold matching for the new information, a change is requested for addition of information to the DAR. Request for change of information in the KR is forwarded to the Change Management module.

Interoperability within the system is achieved by using the standard structures of $H L 7$ (www.hl7.org) and openEHR (www.openehr.org). These are the most frequently used approaches in most Hospital Information Management Systems (HIMS). For the baseline structure $\quad S N O M E D \quad C T$ (http://www.connectingforhealth.nhs.uk/systemsandser vices/data/uktc/snomed) is used.

\subsection{Change Management}

The CM module facilitates the dynamic nature of the DDSS. It is responsible for the KR evolution with the advancement of domain knowledge (including nutritional and dietary information), modifications from experts, with migrating information, with change in patient behavior, and with the social interaction and patient experience. The CM modules accepts change requests, implements the change and propagates the changes to the dependent component for the purposes of minimize their effects. Implemented changes are logged in the Change Log for later audit purposes

\section{Algorithm ChangeManagement (Request):}

This algorithm has multiple sub modules that are responsible for different sub tasks like DAR or Rule Base Recovery. These are just called from the main algorithms.

Input: Request (change or audit or recovery) request from user or system agent. Dynamic Activity ontology $O$

Output: Execution of request.

$/ *$ Perform these steps if the request is change request */ If Request $=$ ChangeRequest then

$/ *$ Receive change request and formally represent the request $* /$ ChangeRequest $\leftarrow$ getChangeRequest(Request) ChangeRequest $\leftarrow \quad$ ChangeRequest .formulate(ChangeRequest, $O$ )

$/ *$ Resolve syntactic and semantic conflicts because of change request $*$ /

ChangeRequest $\leftarrow$ reform(ChangeRequest,$O)$

/* Implement the requested changes, propagate the changes and $\log$ the implemented changes $* /$ $O \leftarrow$ implement (ChangeRequest) propogate(ChangeRequest, RuleBase) EndIf logChangeRequest(ChangeRequest, ChangeLog)

* Call recovery module if the request is for recovery */ recovery.execute(ChangeRequest)

$/ *$ Call audit module if the request is for audit */ audit.execute(ChangeRequest) End Algorithm-2, Change Management combined algorithm. 
including change recovery, for collaborative simultaneous manipulation, and for understanding the KR evolution history. The Change Log ontology is developed for change logging. Algorithm 2 is the implementation details for the change management.

\section{Implementation and Results}

The DDSS is deployed on the Cloud to support patients for their daily life activities, nutritional and dietary intakes, and to promote social interaction. A Smart Phone is used as the main input source for the system. Nine different sensors (i.e., GPS, Accelerometer, Camera, Proximity, Light, Time, Gyroscope, Bluetooth, and MIC) and SMS service are used to collect patient activity and experience information. All the experiments presented in this section have been conducted on a local machine with 4 GB memory and a $2.67 \mathrm{GHz}$ Quad Core processor. Jena2, Protégé, Protégé-OWL, Arq and Pellet 3.4 (for inference) have been used. Accuracy of recommendations generated by the DDSS is dependent on the reported activities. For inference, relevant information is extracted from the DAR using SPARQL queries (an instance is given in Figure 6).

"SELECT ?activityName ?hasConsequentAction ?performedBy ?time ?performedAtLoc " "WHERE $\{<"+$ strNS + strActivity + "><" + strNS + "hasName> ?activityName." +

"<" + strNS + strActivity + "><" + strNS + "hasConsequentAction $>$ ? hasConsequentAction ." + "<" + strNS + strActivity + "><" + strNS + "performedBy $>$ ?performedBy." +

$"<"+$ strNS + strActivity + " $><"+$ strNS + "performedAtTime ? ?time ." +

"OPTIONAL \{<" + strNS + strActivity + "><" + strNS + "performedAtLoc > ?performedAtLoc\}\}";

Figure 6, SPARQL query to extract information form DAR.

Table 1 Performance and accuracy comparison for matching system selection

\begin{tabular}{|c|c|c|c|c|}
\hline System & \multicolumn{2}{|c|}{ HL7 $7_{\text {v } 2.5}$ vs. openEHR } & \multicolumn{2}{|c|}{ HL7 $_{\text {v } 3.0}$ vs. openEHR } \\
\hline & Time & Match & Time & Match \\
\hline Falcon $[8]$ & $0.23 \mathrm{~ms}$ & 16 instances & $0.26 \mathrm{~ms}$ & 16 instances \\
\hline Lily [17] & $0.31 \mathrm{~ms}$ & 15 instances & $0.34 \mathrm{~ms}$ & 17 instances \\
\hline $\begin{array}{l}\text { AgreementMaker } \\
{[2]} \\
\end{array}$ & $0.13 \mathrm{~ms}$ & 16 instances & $0.15 \mathrm{~ms}$ & 18 in \\
\hline
\end{tabular}

To support the match making process for activities against the knowledge from the DAR, an ontology matching system is used. Different ontology matching systems (i.e., Falcon [8], Lily [17], and AgreementMaker [2]) were tested on HL7 and openEHR ontologies for their performance and accuracy (refer to Table 1). Based on the results, AgreementMaker is selected and used for the match making process within the proposed system. It is used in the SLDI module to calculate semantic affinity.

DDSS is tested on the data set of [10] with an increasing set of activities. The results found are encouraging. As a variety of sensors are used for reporting activities, it makes the decision making difficult to only be based on the matched activities from the KR. Results of the match making alone is not enough for recommendation generation. It is filtered with the rules from the Rule-base to make appropriate personalized recommendations (refer to Figure 7 for examples of the rules).

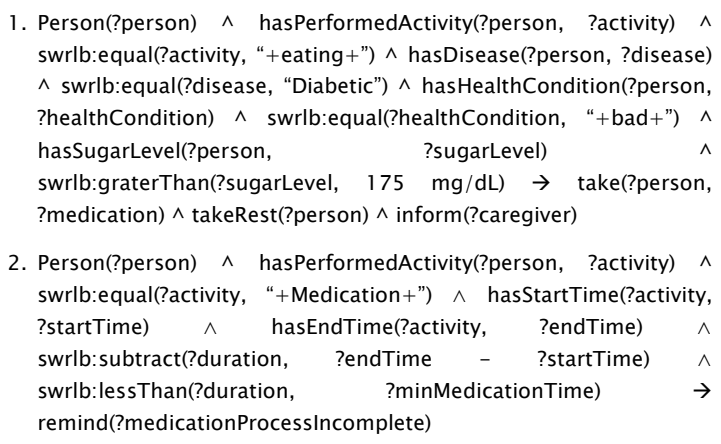

Figure 7. Examples of rules in SWRL format. First rule is a check on the blood sugar level of a patient and the second rule is a check on the process of taking medication.

The CM module is implemented and results of its change listening module are compared with ChangesTab (a Protégé plug-in). The proposed module showed better results than ChangesTab in terms of detecting the number of changes applied to the otology. Figure 8 shows the results of the proposed component against the ChangesTab. The ChangesTab of Protégé detected 28 changes; whereas, the proposed CM module of the DDSS detected 32 changes and has subsequently proved to be a better approach. The reason for missing the 3 changes are that Protégé does not trigger change events for certain types of changes (for example range deletion of Data Type property). Hence, those changes are missed. To make complete recovery from one state of the DAR ontology to another state depends on the detail level logging of all the changes between the two states being considered. As shown in Figure 8 the proposed system has missed 3 changes. To capture and log these missed changes and achieve accurate recovery, the difference() function of the Model class from Jena API is used. Based on this the missed changes are captured and recorded using the difference() function.

Match making and rule based filtering are the main components of the DDSS. They generate appropriate recommendations for patients' daily nutritional and dietary intakes, physical activities, and social interactions. Due to the evolving domain knowledge and dynamic user behavior, the CM continuously updates system's KR that helps the DDSS to generate up to date and personalized recommendations. 


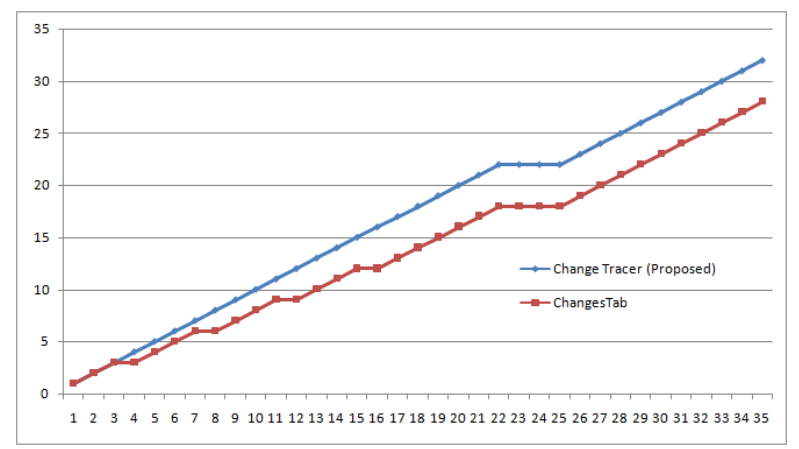

Figure 8, Change Listing with CM of DDSS vs. ChangesTab of Protégé.

\section{Conclusions}

Up to date nutrition related, physical activities related, and social interaction related recommendation generation is achieved in this research. As the domain knowledge constantly grows with changing expert perception, and changing user behavior/experience; it is very important for a system to adapt to the dynamic situation and respond accordingly. The proposed system learns from the environment and responds back appropriately. The facility for information exchange and migration is also achieved by implementing the globally available standards. Results following preliminary investigations have shown encouraging results.

In healthcare systems both performance and accuracy are both important factors to take into consideration. Improved accuracy of generating personalized recommendations will be the main focus for future work.

\section{Acknowledgment}

This work was supported by the National Research Foundation of Korea (NRF) grant funded by the Korea government (MEST) (No. 2011-0030823).

\section{References}

[1] L. Chen, C. Nugent, H. Wang, "A Knowledge-Driven Approach to Activity Recognition in Smart Homes," Knowledge and Data Engineering, IEEE Transactions on, vol. 24, no 6, pp. 961-972, 2012.

[2] I. F. Cruz, F. P. Antonelli, and C. Stroe. "AgreementMaker: Efficient Matching for Large RealWorld Schemas and Ontologies". PVLDB, 2(2):15861589, 2009.

[3] K. Du, D. Zhang, X. Zhou, M. Mokhtari, M. Hariz, and W. Qin. "HYCARE: A hybrid context-aware reminding framework for elders with mild dementia", ICOST 2008.
[4] European Innovation Partnership on Active Ageing, http://ec.europa.eu/information_society/activities/einclu sion/deployment/ahaip/consultation/index_en.htm

[5] I. Fatima, M. Fahim, D. Guan, Y. K. Lee, and S. Y. Lee, "Socially Interactive CDSS for u-Life Care", The 5th ACM International Conference on Ubiquitous Information Management and Communication (ACM ICUIMC 2011), Seoul, Korea, February, 2011.

[6] T. Gao, C. Pesto, L. Selavo, Y. Chen, J. Ko, J. Lim, A. Terzis, A. Watt, J. Jeng, B. Chen, K. Lorincz, M. Welsh, "Wireless Medical Sensor Networks in Emergency Response: Implementation and Pilot Results", The 2008 IEEE International Conference on Technologies for Homeland Security, May, 2008.

[7] K. Henricksen, and J. Indulska, "Modelling and Using Imperfect Context Information". In Proceedings of the Second IEEE Annual Conference on Pervasive Computing and Communications Workshops, Washington DC, March 14 - 17, 2004.

[8] W. Hu, and Y. Qu. Y. "Falcon-AO: A practical ontology matching system". Journal of Web Semantics. 6, 3, pages 237-239, 2008.

[9] A. M. Khattak, L. T. Vinh, D. V. Hung, P. T. H. Truc, L. X. Hung, D. Guan, Z. Pervez, M. Han, S. Y. Lee and Y. K. Lee, "Context-aware Human Activity Recognition and Decision Making", 12th International Conference on e-Health Networking, Application Services(IEEE HealthCom 2010), Lyon, France, July 1-3, 2010.

[10] A. M. Khattak, Z. Pervez, S. Y. Lee, and Y. K. Lee. "Intelligent Healthcare Service Provisioning Using Ontology with Low-Level Sensory Data". KSII Transaction on Internet and Information Systems, vol. 5, no. 11, November 2011.

[11] N. Marmasse, C. Schmandt, "Location-aware information delivery with commotion". The 2nd International symposium on Handheld and Ubiquitous Computing, Bristol, England, pp. 157-171 (2000).

[12] T. Plotz, P. Moynihan, C. Pham, and P. Olivier, "Activity recognition and healthier food preparation", Activity recognition in intelligent pervasive environments, Atlantis Press, pp. 311-327, 2011.

[13] T. Sohn, K. Li, G. Lee, I. Smith, J. Scott, W. Griswold, "Place-its: A study of location-based reminders on mobile phones". In: Ubicomp, pp. 232-250, 2005.

[14] World Health Organization, Active Ageing makes the difference,

http://www.who.int/ageing/publications/alc_embrace20 01_en.pdf

[15] World Health Organization, Keep fit for life: Meeting the nutritional needs of older persons, pp.1-83, 2002.

[16] F. Wang and K. J. Turner. "An Ontology-Based Actuator Discovery and Invocation Framework in Home Care Systems," 7th International Conference on Smart Homes and Health Telematics, pp. 66-73, LNCS 5597, Springer, Berlin, June 2009.

[17] P. Wang and B. Xu. "Lily: Ontology alignment results for oaei 2009". Ontology Matching (OM), 2009. 